\title{
C3b-Independent Complement Activation in Ischemia/Reperfusion Mesenteric Tissue Injury in Autoimmune Prone (B6.MRL/lpr) Mice
}

\author{
J. Tofferi, ${ }^{1,2}$ S. Peng, ${ }^{3}$ and C. M. Moratz ${ }^{4}$ \\ ${ }^{1}$ Division of Rheumatology, Walter Reed Army Medical Center, Washington, DC 20889, USA \\ ${ }^{2}$ Department of Rheumatology, San Antonio Military Medical Center, San Antonio, TX 78234, USA \\ ${ }^{3}$ Department of Rheumatology, Fort Belvoir Community Hospital, Fort Belvoir, VA 32060, USA \\ ${ }^{4}$ Department of Medicine, Uniformed Services University of the Health Sciences, 4301 Jones Bridge Road, Bethesda, MD 20814, USA
}

Correspondence should be addressed to C. M. Moratz, chantal.moratz@usuhs.edu

Received 11 June 2012; Accepted 11 July 2012

Academic Editors: P. A. Berg, R. Merino, and B. Stijlemans

Copyright $\odot 2012$ J. Tofferi et al. This is an open access article distributed under the Creative Commons Attribution License, which permits unrestricted use, distribution, and reproduction in any medium, provided the original work is properly cited.

Complement plays a critical role in the development of tissue injury in systemic lupus erythematosus. The B6.MRL/lpr mouse, an autoimmune prone mouse, exhibits accelerated and intensified tissue injury in the ischemia/reperfusion (IR) model. It has been demonstrated in nonautoimmune mice that inhibition of complement attenuates inflammatory tissue injury in IR models. The role of complement is not as clear in the B6.MRL/lpr strain. B6.MRL/lpr-C3 deficient animals are susceptible to injury, but long-term use of C3 inhibitors in B6.MRL/lpr-C3 competent animals restrained the development of nephritis. To clarify the role of complement in the B6.MRL/lpr strain, initial and midpathway inhibitors were evaluated. $\mathrm{C} 1$ inhibition attenuated tissue injury, thrombin deposition, and C5a generation in the B6.MRL/lpr strain. Downstream of C1 inhibition of C3 activation by administration of cobra venom factor suppressed IR injury in immune competent mice, but was not as effective in B6.MRL/lpr mice. C3 levels in both strains were decreased after cobra venom factor treatment; however, C5a generation, thrombin deposition, and tissue injury were observed in the B6.MRL/lpr strain. These studies suggest that in the B6.MRL/lpr autoimmune prone strain $\mathrm{C} 1$ activation leads to C3-dependent and C3-independent pathways of complement activation.

\section{Introduction}

The inflammatory response to pathogens is a critical protective mechanism of the immune system. In this process, the pathogen is recognized as nonself and harmful; an innate immune response is initiated; cells migrate into the infected site to clear the pathogen; interactions between the innate and adaptive response and other cues govern the extent of inflammation. Other cues govern the resolution of the immune response [1-4]. The initiation, maintenance, and resolution of immune-mediated inflammation are tightly regulated by numerous host mechanisms such as self-tolerance, signal transduction regulation, and apoptotic clearance of cellular fragments. The breakdown in regulating the cellular mechanisms listed above results in deleterious conditions for the host; often leading to the emergence of an autoimmune condition. One such systemic autoimmune disease, Systemic Lupus Erythematosus (SLE), has a complex pathogenesis. Clinically, SLE entails recurrent unpredictable exacerbations and remissions as well as widespread and diverse tissue and vascular lesions with renal disease (lupus nephritis) as a major cause of morbidity and mortality. The lupus prone mouse model B6.MRL/lpr develops an early onset autoimmune disease similar to human SLE with most mice dying from glomerulonephritis $[5,6]$. There is no single specific cause or gene abnormality resulting in SLE. It is a result of a complex overlay of multigene susceptibilities and environmental factors. However at the bottom of its pathology is a defect in tolerance, T-cell function, autoantibody production, immune complex deposition, and hyperinflammatory responses and vascular lesions. One biological pathway that may be of importance in regulating many of the conditions listed above is the complement system. 
Ischemia/reperfusion (IR) models of tissue injury are characterized by depletion of oxygen to tissue via lack of blood flow, followed by reoxygenation with return of blood flow to affected tissues. The cellular mechanisms that result in tissue injury and destruction occurring after reperfusion are divided into two phases: the acute and subacute phase. The acute phase is characterized by oxidative tissue injury resulting in activation of intracellular damage pathways, nitric oxide overproduction, inducible nitric oxide synthetase, and initiation of antibody-mediated complement activation [7-10]. The subacute phase is characterized by leukocyte infiltration and macrophage activation and histological detectable tissue injury [11-13]. Much of this damage is caused by the complement system. In addition to facilitating direct cell lysis, activation of the complement cascade increases phagocytosis, recruits inflammatory molecules, and instructs the adaptive response to produce an appropriate humoral response. Local injury as a consequence of mesenteric IR tissue injury has been well characterized in mouse models by several groups using multiple endpoints (histopathology, neutrophil infiltration, permeability index). It has been shown in the mesenteric IR model that complement inhibitors reduce tissue injury [14-16]. Other studies have shown that $\mathrm{C}^{-/-}$and $\mathrm{C} 4^{-/-}$ mice are resistant to complement-induced tissue injury [17, 18]. Furthermore, studies in the mesenteric IR model have demonstrated that injury is accelerated and intensified in the B6.MRl/lpr autoimmune strain [19]. However, C3 deficient B6.MRL/lpr mice are not resistant to injury [20], suggesting C3 is not essential for tissue injury. Conflicting with studies utilizing the C3 deficient/B6.MRL.lpr strain are studies using C3 inhibitors in competent B6.MRL/lpr animals. The inhibitor studies in C3 competent B6.MRL/lpr animals have demonstrated long-term use of inhibitors of C3 are effective in attenuating tissue injury and restrains the development of nephritis $[21,22]$. This dichotomy of complements role is not restricted to the specific animal model, depressed levels of C3 in SLE patients are documented, as is complement involvement in tissue injury [23-27].

To clarify the role of complement in mesenteric IR tissue injury in the B6.MRL/lpr strain, initial and midpathway inhibitors were evaluated. We utilized the $\mathrm{C} 1$ esterase inhibitor to target the initiation of the complement cascade and then used treatment with cobra venom factor (CVF) to deplete $\mathrm{C} 3$, the midcascade convergence point. $\mathrm{C} 1$ esterase inhibition attenuated tissue injury. However, depletion and stabilization of C3b through the administration of CVF attenuated IR injury in immune competent, but was not as effective in the in B6.MRL/lpr mice. In the B6.MRL/lpr mice, despite C3 depletion by CVF, generation of C5a was detected in the injured tissue. These studies suggest that in the B6.MRL/lpr autoimmune prone strain, C1-mediated complement activation proceeds through C3-dependent and -independent pathways to result exacerbated tissue injury.

\section{Materials and Methods}

2.1. Animals. B6.MRL/lpr autoimmune mice and $\mathrm{C} 57 \mathrm{Bl} / 6$ mice were obtained from The Jackson Laboratory (Bar
Harbor, ME) and maintained in the Uniformed Services University animal facility until they were 5 months of age. By 5 months of age the autoimmune phenotype is fully established in the B6.MRL/lpr animals. Animals in this study were maintained in accordance with the guidelines of the Laboratory Animal Medicine Department/IACUC of Uniform Services University of the Health Sciences animal facility.

2.2. Mesenteric Model of IR. The mesenteric IR injury model was performed as previously described [28-30]. Briefly, mice were anesthetized with ketamine and xylazine administered interperitoneally. The animals were placed on a water circulating heating pad to maintain a $37^{\circ} \mathrm{C}$ body temperature. The animals were subjected to a midline laparotomy, a small vascular clamp (Roboz Surgical Instruments, Rockville, MD, USA) was applied for 30 minutes to the superior mesenteric artery (SMA). After release of the clamp, the animals were monitored during the one hour reperfusion period. Sham animals were subject to the same surgical intervention, except the SMA was not clamped. Mice were kept anesthetized for the duration of the experiment. Additional ketamine and xylazine were injected intramuscularly before euthanasia. After euthanasia, samples of the small intestine, approximately $10 \mathrm{~cm}$ distal to the gastroduodenal junction, were taken. All procedures were reviewed and approved by the institute's Animal Care and Use Committee. In the $\mathrm{C} 1$ esterase inhibitor studies, purified human Esterase inhibitor C-1 (Sigma-Aldrich, Cat \#C2412, Dosage used was $0.004 \mathrm{mg} / 100 \mathrm{uL}$ or $150 \mathrm{ng} / \mathrm{g}$ body weight) was injected 5 minutes prior to reperfusion by tail vein injection. In the CVF depletion studies, animals were injected interperitoneally with $200 \mathrm{ng} / \mathrm{g}$ body weight dose of CVF (Naja naja Kaouthia Cat\# 064K4000, Sigma-Aldrich Chemical company) $24 \mathrm{hrs}$ prior to use in the IR procedure.

2.3. Histology. Formalin-fixed paraffin blocks of intestine and lung tissue were made from tissue harvested immediately after euthanasia and fixed in $10 \%$ buffered formalin phosphate. The blocks were sectioned transversely in $7 \mathrm{um}$ sections and stained with hematoxylin and eosin (H\&E). Sections from successive tissue depths were cut for each tissue and stained.

2.3.1. Immunofluorescent Staining of Tissue Sections. The Immunofluorescence staining for either $\mathrm{C} 3 \mathrm{~b}, \mathrm{IgG}$, or C5a was performed on sections fixed for $2 \mathrm{~h}$ in cold $4 \%$ paraformaldehyde in PBS before transfer to PBS for preservation in OCT and preparation of frozen sections. Transverse sections of intestinal tissue were cut and prepared for staining. Frozen slides were dried at room temperature, then fixed in cold acetone for $10 \mathrm{~min}$, after the sections were dried again, slides were washed with PBS and blocked for $30 \mathrm{~min}$ with $2.5 \%$ bovine serum albumin solution in PBS. Slides were then incubated with various antibodies (goat anti-mouse C3-FITC (Cappel laboratories); anti mouse IgG-TRITC (Jackson Immunoresearch); rat anti-mouse C5a-biotin with Streptavidin-PE detection (BD Biosciences, San Jose, CA); 
antithrombin (Santa Cruz, Biotechnology) with Donkey anti-goat IgG-FITC detection (Jackson Immunoresearch)). Finally, slides were mounted with ProLong Gold antifade, counterstained with DAPI ( $4^{\prime}, 6^{\prime}$-diamidino-2-phenylindole; Invitrogen, Carlsbad, CA, USA), and observed with an Olympus Leica A80 fluorescence microscope. Recorded digital images were processed using Image J software $(\mathrm{NIH}$ Bethesda, MD, USA) or Adobe Photoshop CS4 software.

2.4. Intestinal Injury Scoring. Intestine was scored for injury with a point scoring system (0 to 6 points) as previously described [30]. Briefly, zero represented a normal villi; 1 point for edema; 2 points for edema, goblet cells, and missing Gugenhemims' spaces; 3 points for sporadic disruption of the epithelial cell layer; 4 points for villi with complete disruption of the outer epithelial layer with an exposed but intact lamina propria; 5 points for disrupted epithelial layer, lamina propria, and vascular network; 6 points for villi that display hemorrhage or that are denuded.

2.5. Analysis of C3 Hemolytic Activity in Serum. The detection of complement C3 in serum was performed using a modified total hemolytic, $\mathrm{CH} 50$, complement activity assay [31]. The blood was collected by cardiac puncture at the end of the reperfusion time and collection tissue samples. The appropriate series of dilutions of test sera were added into a series of reaction wells (96-well plate assay format) containing Gelatin Veronal buffer (Sigma-Aldrich), C3-depleted human sera (QUIDEL), and sheep red cells sensitized by rabbit anti-sheep red cell antibody (SigmaAldrich). After incubation at $37^{\circ} \mathrm{C}$ for $1 \mathrm{~h}$, the plates were centrifuged to pellet unlysed cells. $100 \mathrm{uL}$ of the supernatant for each well was transferred to a new well and the optical density of supernatant was measured at $415 \mathrm{~nm}$.

2.6. Statistical Analysis. Data are presented as mean \pm SEM with difference between groups considered significant when $P<0.05$. The data was analyzed by one-way analysis of variance with post hoc analysis (Graph Pad/Instat Software, Inc. Philadelphia, PA, USA).

\section{Results}

3.1. Analysis of C1 Inhibition in the IR Model of Inflammatory Tissue Injury. Prior studies have demonstrated that complement activation and secondary inflammation are responsible for tissue injury in the IR model of inflammatory tissue injury. In nonautoimmune strains of mice it has been shown that antibody and complement activation are essential for tissue injury in the IR model $[16,17,30]$. Specifically, C1 esterase was shown to inhibit mesenteric IR injury in nonautoimmune mice [16]. To determine if abnormal complement activation contributes to the accelerated and intensified tissue injury in the mesenteric IR model, C1 esterase was used to inhibit complement activation in the initial step of the complement cascade. As in previous studies, $\mathrm{C} 1$ esterase reduced induced tissue injury in the normal C57B.6 mice as detailed in Figure 1(a). The graph represents a scoring system of tissue structure integrity. Each incremental increase in score number indicates increasing levels of structural impairment. In sham animals the epithelial barriers, endothelium lining of circulatory vessels, and lamina propria of the villi are normal. With injury the score increases from tissue edema (level 1) to complete destruction of the villi (level 6). C1 esterase treatment of C57B.6 animals reduced the injury to the intestinal tissue to a mild injury and edema, with intact integrity of the villous structure, as illustrated in Figure 1(b). In the autoimmune strain B6.MRL/lpr, treatment with $\mathrm{C} 1$ esterase also attenuated tissue injury. Hematoxylin and eosin (H\&E) staining of intestinal sections are used to score injury levels. In Figure 1(b) the H\&E stained section illustrated the condition of the intestinal tissue from Sham, IR, and IR with inhibitor treatment.

\subsection{Analysis of C3 Depletion in IR Model of Inflammatory} Tissue Injury. Complement component C3 is downstream in the cascade from $\mathrm{C} 1$ and can be activated by the classical (C1q dependent), alternative, and Mannose Binding lectin complement initiation pathways, It is also the convergence point in the cascade leading to $\mathrm{C} 5$ activation and subsequent C5-9 membrane attack complex formation. In these studies we utilized pretreatment with cobra venom factor (CVF) to deplete C3 from the circulation. B6.MRL/lpr mice displayed significant intestinal IR damage despite depletion of complement with CVF, Figure 2(a). The degree of injury was comparable to the injury in these mice without complement inhibition. In contrast, depletion of C3 in nonautoimmune C57BL/6 mice effectively abrogated tissue damage in the IR model. The H\&E images in Figure 2(b), illustrates the extent of injury for each treatment group. The tissue integrity of intestinal tissue for the B6.MRL/lpr-CVF-treated group was severely compromised with breakdown of the epithelial cell barrier, disruption of the vasculature, as well as dissociation of the lamina propria. On the other hand, in the C57B/6CVF-treated group tissue injury was limited to edema and mild agitation of the epithelial barrier. To determine if complement C3 was depleted from the circulation, serum from animals in each treatment group was assayed in a $\mathrm{C} 3 \mathrm{H} 50$ assay measuring total hemolytic activity of $\mathrm{C} 3$ in the serum. The graph in Figure 3(a) shows that C3 hemolytic activity is detected in the serum of B6.MRL/lpr BSA-IR animals but not in the serum of B6.MRL/lpr CVF-IR animals, indicating that $\mathrm{C} 3$ was absent from the circulation in the B6.MRL/lpr animals. Immunofluorescence staining of intestinal tissue sections from Sham and Control-IR indicate low C3 (green) staining and moderate IgG (red) staining in the sham animals, as shown in the left panel of Figure 3(b). Abundant C3 deposition and IgG staining is detected in the control-IR group, with extensive colocalization (yellow), as shown in the center panel. However in the right side panel, IgG is detected but minimal C3 is detected in the CVF-IR B6.MRL/lpr group.

3.3. Detection of C5a in C3-Depleted Animals. To clarify the role of complement activation in the injury detected in the CVF-IR-treated B.6/MRL/lpr animals, downstream complement activation components were assessed. The structural 

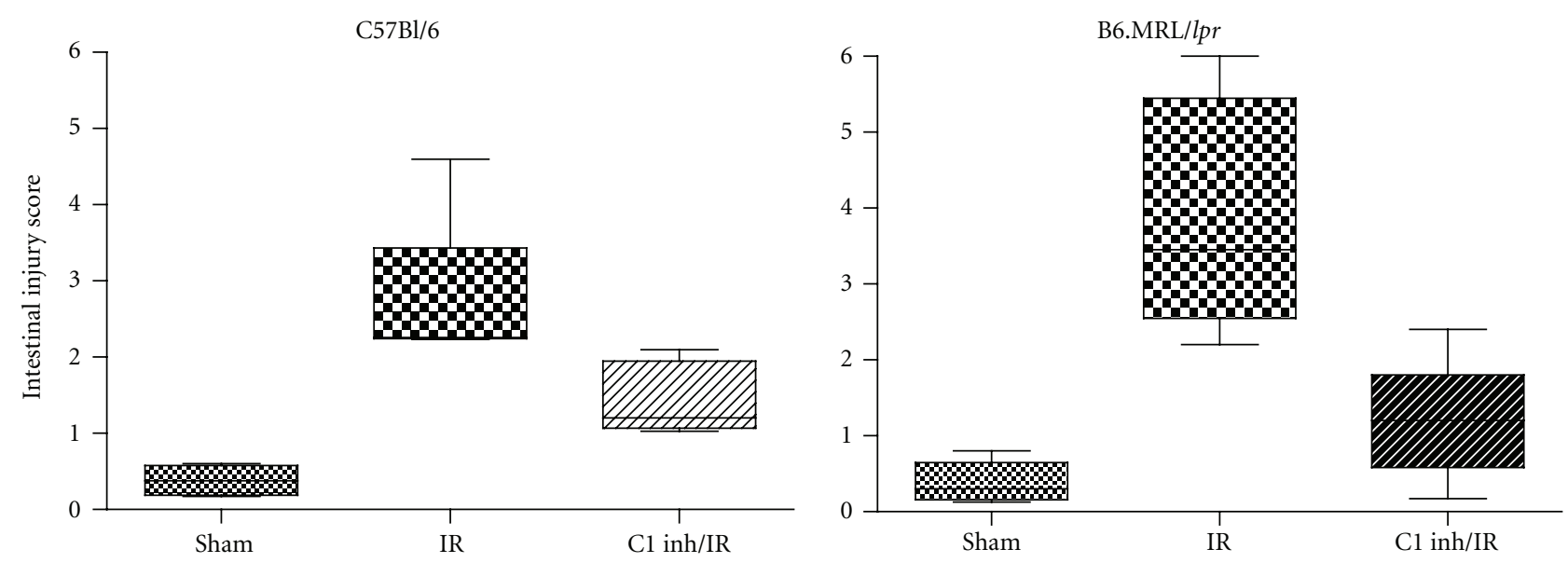

(a)
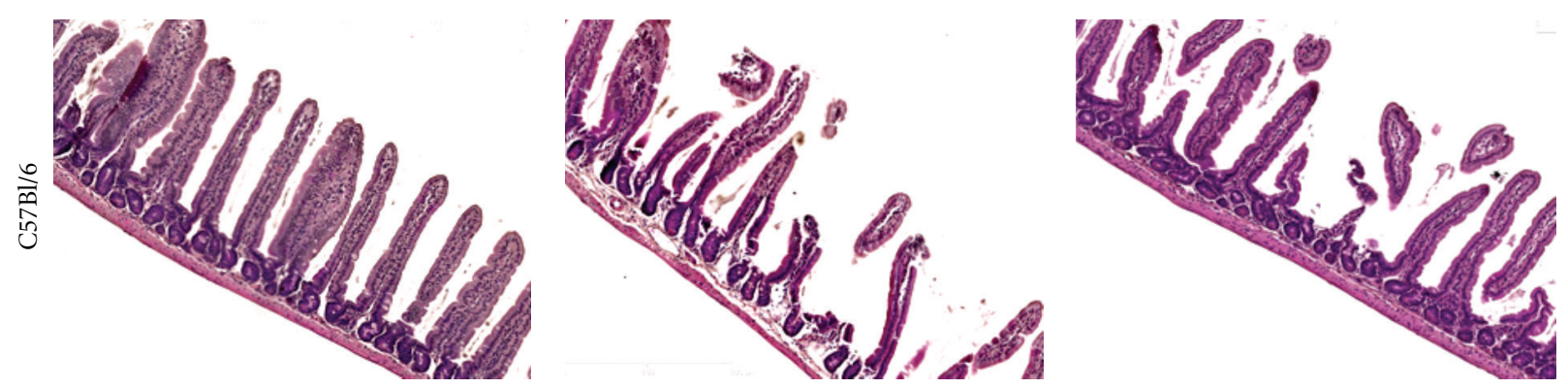

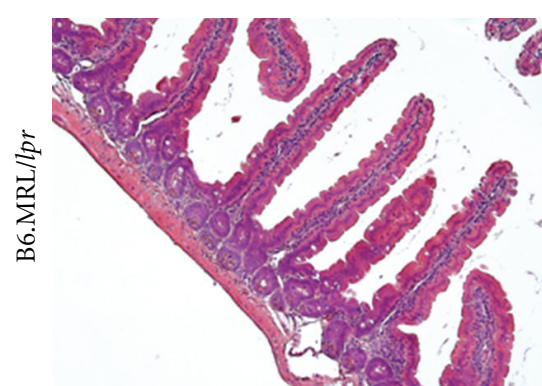

Sham

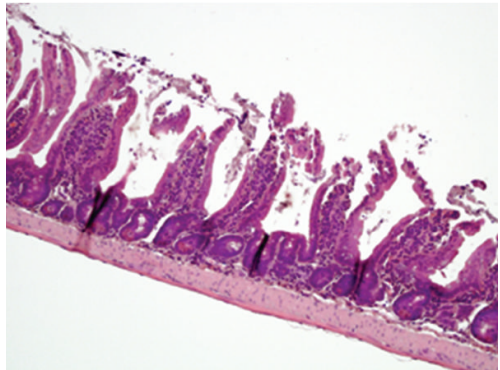

IR

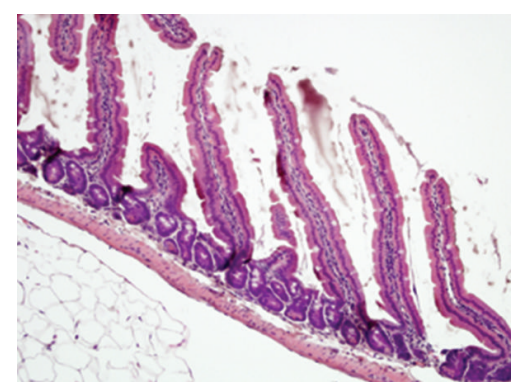

$\mathrm{C} 1 \mathrm{inh} / \mathrm{IR}$

(b)

FIGURE 1: Analysis of C1 inhibition in the IR model of inflammatory tissue injury. H\&E-stained transverse intestinal sections from inhibition studies were evaluated. (a) Graphical representation of the injury scores form the C1 esterase inhibition studies are shown. By one-way ANOVA testing, specifically the Kruskal-Wallis test, indicated significance between groups in both the B6.MRL/lpr group $(P<0.0005)$ and the C57Bl/6 group $(P<0.001)$. Subsequent analysis between groups within each strain with Dunn's multiple-comparison test indicated that there is a significant difference between the sham and IR group but not the sham and C1 inhibition group. Analysis is performed on five animals per treatment group. (b) H\&E images illustrate the representative injury level detected in each group.

tissue damage seen in the C3-depleted autoimmune strain was typical of complement induced injury in this model. Based on current reports of alternative mechanisms of C5 activation, tissue sections from animals in each treatment group were stained for the activated split product $\mathrm{C} 5 \mathrm{a}$. As expected in B6.MRL/lpr sham animals no activated C5a split product was detected (panel 1 in Figure 4(a)) but it was detected in control-IR tissue (panel 2). Inhibition with $\mathrm{C} 1$ esterase attenuated the production of $\mathrm{C} 5 \mathrm{a}$ in the B6.MRL/lpr mice as shown in Figure 4(a), panel 3. However
CVF-treatment or C3 depletion in the B6.MRL/lpr mice did not decrease the production of C5a in IR animals, Figure 4(a) panel 4. A possible explanation of how C5a is generated in the CVF treated animals is that thrombin generated during tissue injury could subsequently activate C5. Figure 4(b) illustrates, by immunofluorescence staining of tissue sections, the presence of thrombin in injured tissues. In the control IR and CVF-treated IR B6.MRI/lpr animals thrombin is detected, but thrombin is not present in $\mathrm{C} 1$ esterase-treated IR B6.MRL/lpr animals. 


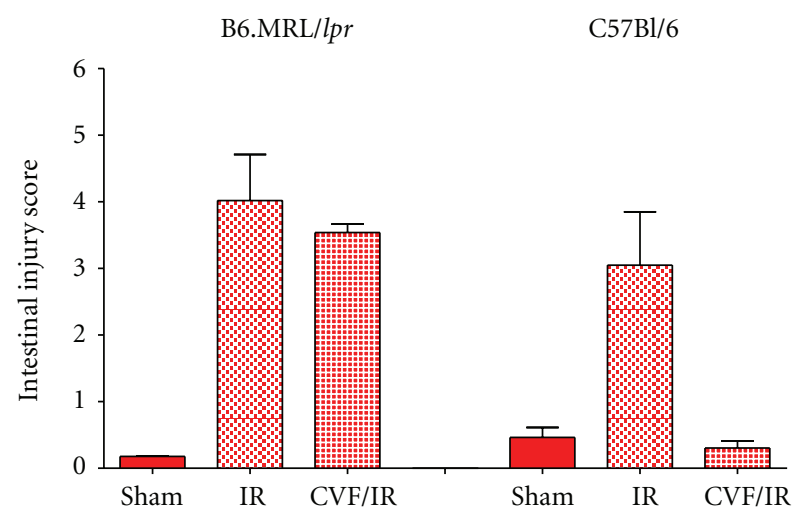

(a)

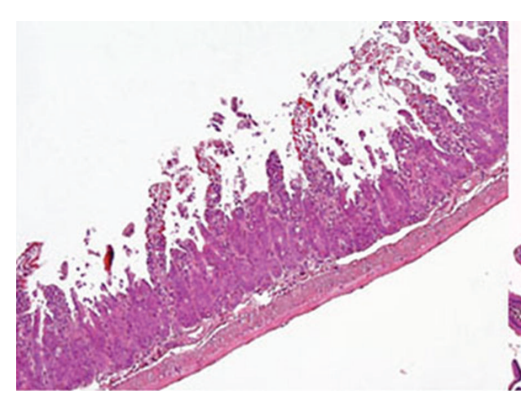

B6.MRL/lpr

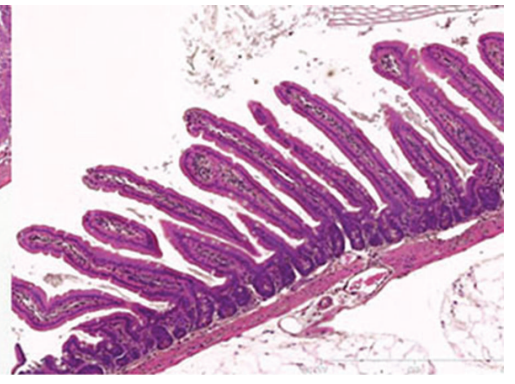

$\mathrm{C} 57 \mathrm{Bl} / 6$

(b)

FIGURE 2: Analysis of C3 depletion in IR model of inflammatory tissue injury. Injury scores generated from H\&E stained transverse sections of intestinal tissue are analyzed. (a) Injury scores for treatment groups in each strain are graphed. Five-month old B6.MRL/lpr mice displayed significant intestinal IRI damage despite depletion of complement with CVF. The degree of injury was comparable to the injury that occurs in these mice without any complement inhibition. In contrast, depletion of C3 in CVF treated C57BL/6 mice effectively abrogated tissue damage in the IRI model. By one-way ANOVA testing, specifically the Kruskal-Wallis test indicated significance between treatment groups in both the B6.MRL/lpr set $(P<0.004)$ and the $\mathrm{C} 57 \mathrm{Bl} / 6$ set $(P<0.03)$. Data represents analysis of 5 animals per group. (b) H\&E images illustrate the representative injury level detected in the CVF depleted IR groups of C57Bl/6 and B6.MRL/lpr strains.

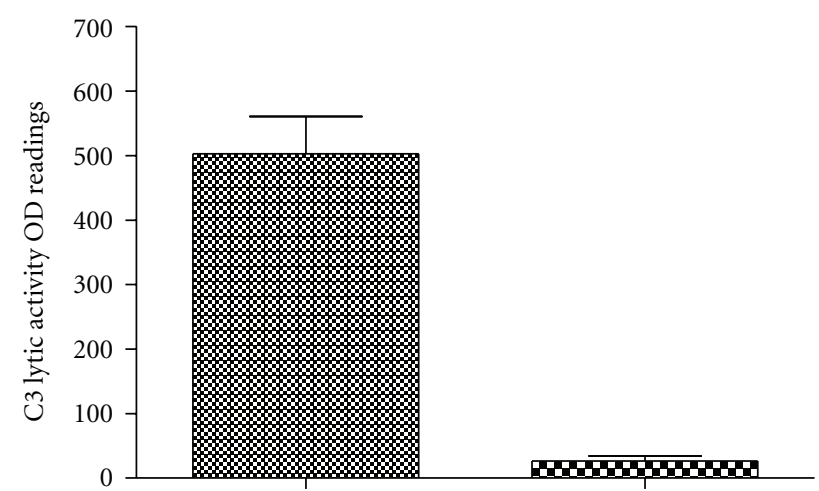

BSA/IR
CVF/IR

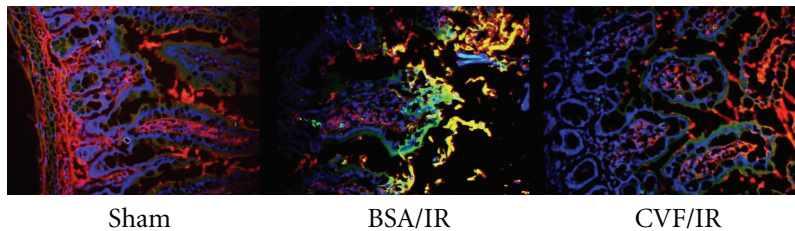

(b)

(a)

FIgure 3: Analysis of C3 hemolytic activity in serum. Confirmation that C3 was depleted. (a) Blocking of the complement cascade at C3 was confirmed with serum measurements of $\mathrm{C} 3$ in a $\mathrm{C} 3 \mathrm{CH} 50$ assay. Data is based on analysis of three animals per group. (b) Immunofluorescent staining of intestinal tissue section's from sham, control-IR, and CVF-IR-depleted animals indicate that low C3 (green) staining and moderate IgG (red) staining is detected in the sham tissue (left panel). High levels of C3 and IgG are detected in the control-IR tissue, with extensive colocalization (yellow), as shown in the center panel. In the right-side panel the extensive colocalization of IgG and C3 is not detected in the CVF-IR B6.MRL/lpr group, however levels similar to the sham (left panel) are detected. 


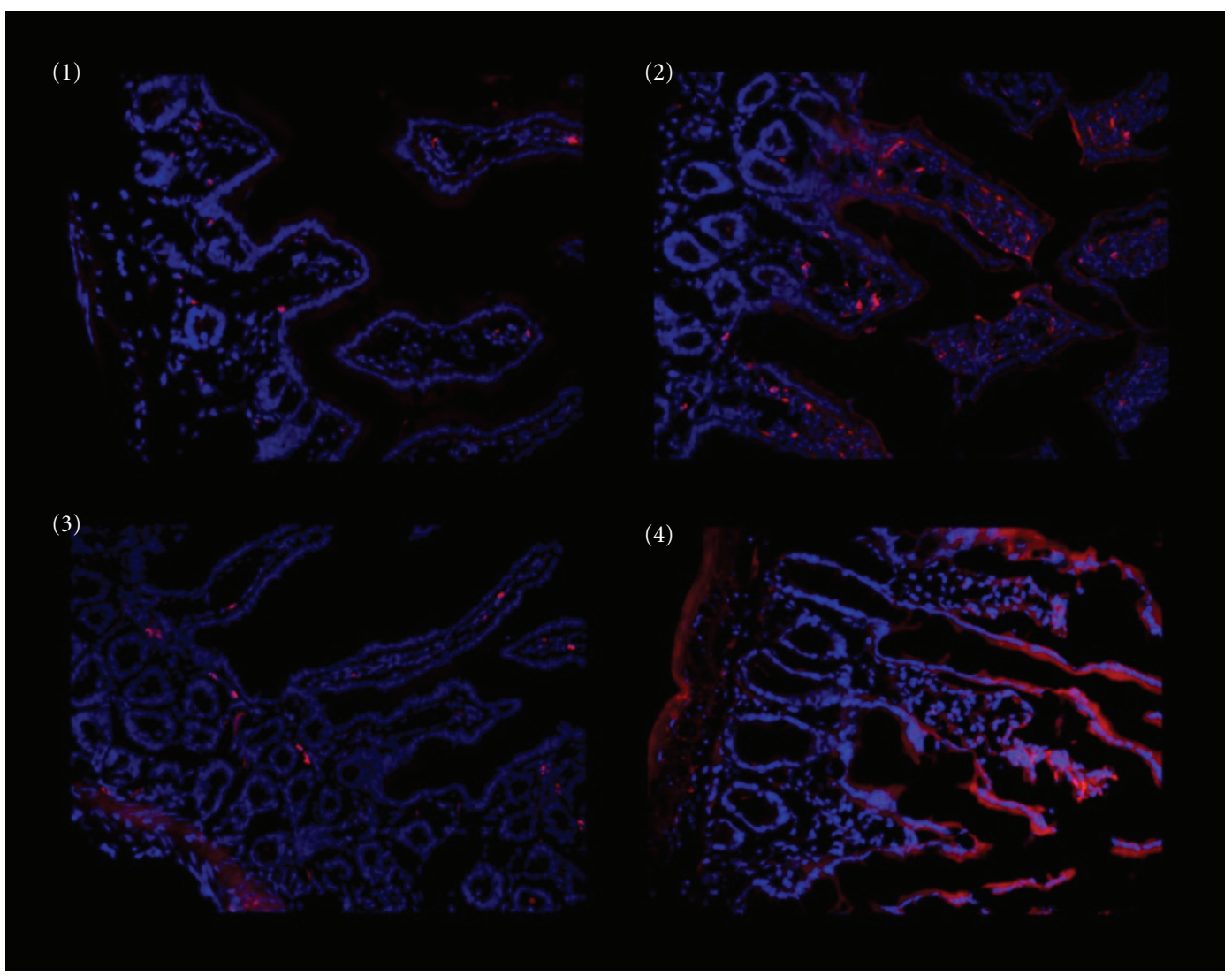

(a)

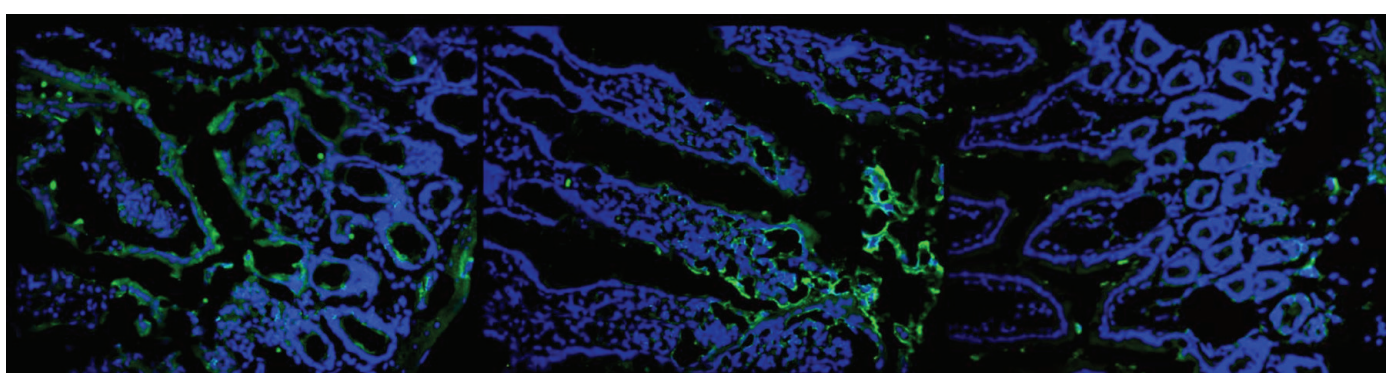

Sham

CVF/IR

$\mathrm{C} 1 \mathrm{inh} / \mathrm{IR}$

(b)

FIGURE 4: Detection of C5a and thrombin in C3 deleted B6.MRL/lpr animals. Images of immunofluorescence staining of transverse intestinal tissue sections are presented. (a) C5a is not detected in panels 1: sham tissue, or panel 3: C1 esterase treated IR tissue. C5a is detected in panel 2: IR tissue and panel 4: CVF treated IR tissue. (b) Thrombin staining is detected in the IR tissue (left panel) and CVF tissue (center panel), but is absent in the $\mathrm{C} 1$ esterase-IR tissue (right panel). The images used are representative images taken from analyses of tissue sections from each animal in the group, with a group size of 5 animals.

\section{Discussion}

Early complement cascade inhibition by $\mathrm{C} 1$ esterase attenuation of tissue injury in the B6.MRL/lpr autoimmune strain confirms that complement activation has an essential role in tissue injury. The C1q downstream targets are platelets as well as intermediates in the thrombin cascades $[32,33]$. Soluble CR1 (sCR1) has been produced by recombinant technology and is being used in phase I human trials.

However, in our study, the amount of tissue injury in the B6.MRL/lpr autoimmune strain was unchanged if C3 was depleted with CVF. Depletion of C3 by CVF in the nonautoimmune $\mathrm{C} 57 \mathrm{Bl} / 6$ strain attenuated tissue injury. The data suggests that in the B6.MRL/lpr autoimmune prone strain there may be a greater reliance on a C3independent pathway for complement activation during acute inflammatory responses. After injury in this model, downstream mediators of inflammation such as IL-6 and IL-1 are produced in both the $\mathrm{C} 57 \mathrm{Bl} / 6$ and $\mathrm{B} 6 . \mathrm{MRL} / \mathrm{lpr}$ animals and inhibition of injury, by T-cell depletion, results in attenuation of these secondary mediators in both strains (unpublished data). In ongoing work, the generation of these 


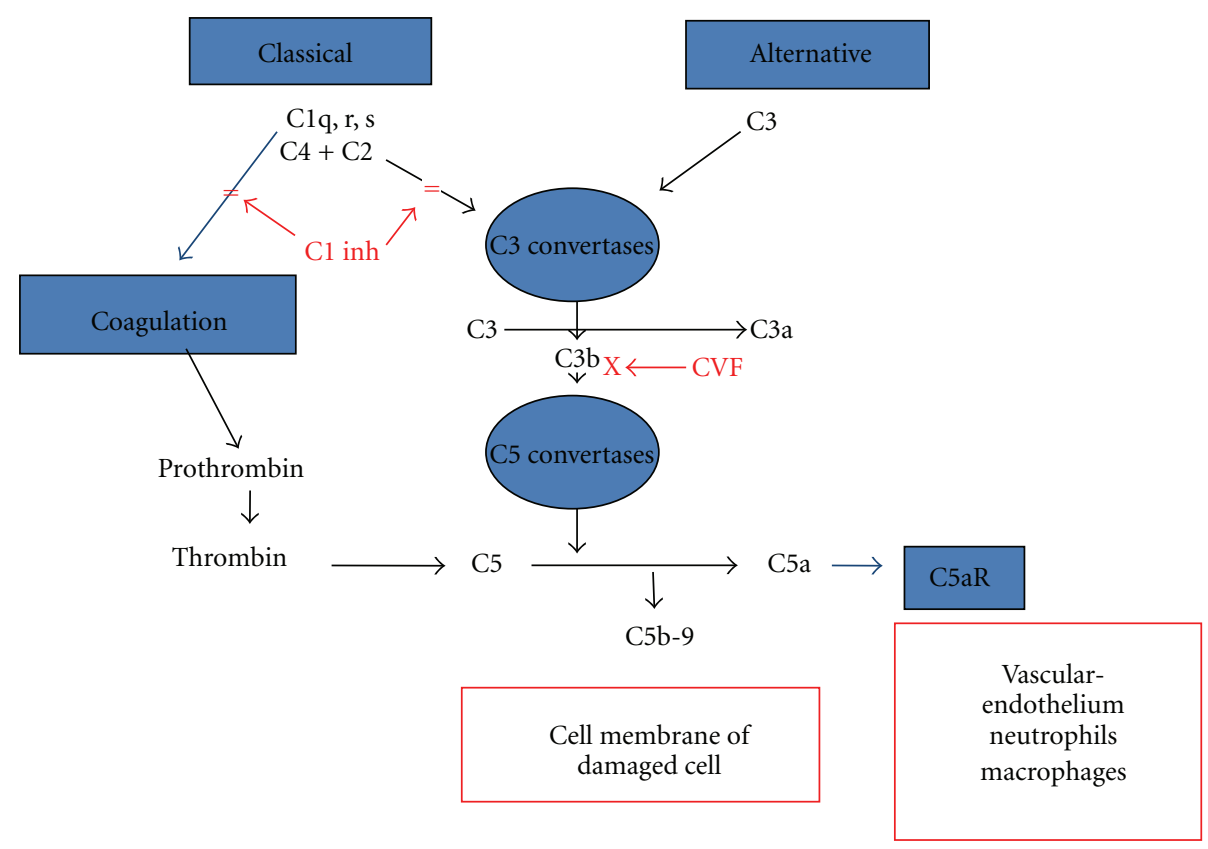

Figure 5: Complement C3-independent tissue injury pathway. A schematic, which depicts a possible alternative interaction between the complement cascade and coagulation cascade, is presented. The cartoon diagrams a possible explanation of the data presented, specifically how the C3 component of the complement cascade can be bypassed to produce activated C5a.

secondary mediators of inflammation after injury is being investigated when complement and coagulation inhibitors are used in the B6.MRL/lpr strain.

Specifically, our findings of thrombin and C5a generation in injured tissue of the C3-depleted B6.MRL/lpr mice implicates the coagulation pathway as part of this alternate amplification loop, as depicted in a model schematic in Figure 5. Several studies have begun to suggest a link between the inflammatory and coagulation pathways [34-37]. It is reasonable to suggest a regulatory link between the complement and coagulation cascades. Both are very similar in their basic mechanisms of activation. Each utilizes a series of components which must be activated in succession. Each pathway has multiple points at which the cascade can be amplified or negatively regulated [36]. Several studies have suggested that thrombin may function as a C5 convertase in the genetic absence of C3 [34]. Others studies have identified this point in the complement cascade as one in which the complement activation influences the coagulation pathway [38-40]. It has been speculated that the physiological conditions determine which pathway dominates and regulates the other. However the specific details or mechanisms have not been elucidated. In our study $\mathrm{C} 1$ esterase inhibition limited tissue injury, the mechanism of action may be due to direct inhibition of downstream mediators of the complement cascade. Alternatively, and perhaps more importantly in the B6.MRL/lpr strain, inhibition of $\mathrm{C} 1$ activation may reduce platelet activation and thrombosis. Signaling by $\mathrm{C} 1 \mathrm{q}$ receptors on platelets has been associated with increased cell adhesion and thrombosis which would increase thrombin generation [41].
The B6.MRL/lpr mouse is inherently hypocomplementemic. Complement deficiency plays a role in autoimmunity. In the human condition of SLE, during flares the complement system is activated, giving rise to partial deficiency or dysfunction due to consumption [33]. Thus, complement deficiency in the autoimmune prone mouse may be the reason the B6.MRL/lpr mouse utilizes the C3-independent pathway in the IR model. Further investigation should be pursued to elucidate the nature of the association between the complement and coagulation pathways in the autoimmune prone mouse. Clinical implications of this association could be quite significant, particularly in the role of anticoagulation in SLE therapy.

\section{Abbreviations}

CVF: Cobra venom factor

IR: Ischemia/reperfusion

SLE: Systemic Lupus Erythematosus

CR1: Complement receptor one

sCR1: Soluble complement receptor one

SMA: Superior mesenteric artery

H\&E: Hematoxylin and eosin

GPCR: G-protein-coupled receptor.

\section{Acknowledgment}

This paper was supported by Medical Research and Material Command Grant W81XWH-06-10486. The opinions contained in this paper are the private ones of the authors and are not to be construed as official policy or reflecting the views of 
the Department of Defense. The costs of publication of this paper were defrayed in part by the payment of page charges. This article must therefore be hereby marked advertisement in accordance with 18 U.S.C. Section 1734 solely to indicate this fact.

\section{References}

[1] A. Mantovani, M. A. Cassatella, C. Costantini, and S. Jaillon, "Neutrophils in the activation and regulation of innate and adaptive immunity," Nature Reviews Immunology, vol. 11, no. 8, pp. 519-531, 2011.

[2] I. Jarchum and E. G. Pamer, "Regulation of innate and adaptive immunity by the commensal microbiota," Current Opinion in Immunology, vol. 23, no. 3, pp. 353-360, 2011.

[3] M. Koeppen, T. Eckle, and H. K. Eltzschig, "The hypoxiainflammation link and potential drug targets," Current Opinion in Anaesthesiology, vol. 24, no. 4, pp. 363-369, 2011.

[4] A. Iwasaki and R. Medzhitov, "Regulation of adaptive immunity by the innate immune system," Science, vol. 327, no. 5963, pp. 291-295, 2010.

[5] F. Furukawa and T. Yoshimasu, "Animal models of spontaneous and drug-induced cutaneous lupus erythematosus," Autoimmunity Reviews, vol. 4, no. 6, pp. 345-350, 2005.

[6] L. Morel, "Genetics of SLE: evidence from mouse models," Nature Reviews Rheumatology, vol. 6, no. 6, pp. 348-357, 2010.

[7] H. de Groot and U. Rauen, "Ischemia-reperfusion injury: processes in pathogenetic networks: a review," Transplantation Proceedings, vol. 39, no. 2, pp. 481-484, 2007.

[8] S. Kokura, R. E. Wolf, T. Yoshikawa, H. Ichikawa, D. N. Granger, and T. Y. Aw, "Endothelial cells exposed to anoxia/ reoxygenation are hyperadhesive to T-lymphocytes: kinetics and molecular mechanisms," Microcirculation, vol. 7, no. 1, pp. 13-23, 2000.

[9] N. F. Cerqueira, C. A. Hussni, W. B. Yoshida, and C. R. Padovani, "Systemic evaluation on ischemia and reperfusion injury of splanchnic organs in rats," Acta Cirurgica Brasileira, vol. 24, no. 4, pp. 290-295, 2009.

[10] N. F. Cerqueira, C. A. Hussni, and W. B. Yoshida, "Pathophysiology of mesenteric ischemia/reperfusion: a review," Acta Cirurgica Brasileira, vol. 20, no. 4, pp. 336-343, 2005.

[11] R. D. Minshall and A. B. Malik, "Transport across the endothelium: regulation of endothelial permeability," in Handbook of Experimental Pharmacology, pp. 107-144, 2006.

[12] M. Phillipson, J. Kaur, P. Colarusso, C. M. Ballantyne, and P. Kubes, "Endothelial domes encapsulate adherent neutrophils and minimize increases in vascular permeability in paracellular and transcellular emigration," PLoS ONE, vol. 3, no. 2, Article ID e1649, 2008.

[13] E. Dejana, E. Tournier-Lasserve, and B. M. Weinstein, "The control of vascular integrity by endothelial cell junctions: molecular basis and pathological implications," Developmental Cell, vol. 16, no. 2, pp. 209-221, 2009.

[14] L. Kulik, S. D. Fleming, C. Moratz et al., "Pathogenic natural antibodies recognizing annexin IV are required to develop intestinal ischemia-reperfusion injury," Journal of Immunology, vol. 182, no. 9, pp. 5363-5373, 2009.

[15] C. Weeks, C. Moratz, A. Zacharia et al., "Decay-accelerating factor attenuates remote ischemia-reperfusion-initiated organ damage," Clinical Immunology, vol. 124, no. 3, pp. 311-327, 2007.

[16] S. Rehrig, S. D. Fleming, J. Anderson et al., "Complement inhibitor, complement receptor 1-related gene/protein y-Ig attenuates intestinal damage after the onset of mesenteric ischemia/reperfusion injury in mice," Journal of Immunology, vol. 167, no. 10, pp. 5921-5927, 2001.

[17] J. P. Williams, T. T. V. Pechet, M. R. Weiser et al., "Intestinal reperfusion injury is mediated by IgM and complement," Journal of Applied Physiology, vol. 86, no. 3, pp. 938-942, 1999.

[18] W. Zhou, C. A. Farrar, K. Abe et al., "Predominant role for C5b-9 in renal ischemia/reperfusion injury," The Journal of Clinical Investigation, vol. 105, no. 10, pp. 1363-1371, 2000.

[19] S. D. Fleming, M. Monestier, and G. C. Tsokos, "Accelerated ischemia/reperfusion-induced injury in autoimmunity-prone mice," Journal of Immunology, vol. 173, no. 6, pp. 4230-4235, 2004.

[20] H. Sekine, C. M. Reilly, I. D. Molano et al., "Complement component $\mathrm{C} 3$ is not required for full expression of immune complex glomerulonephritis in MRL/lpr mice," Journal of Immunology, vol. 166, no. 10, pp. 6444-6451, 2001.

[21] C. Atkinson, F. Qiao, H. Song, G. S. Gilkeson, and S. Tomlinson, "Low-dose targeted complement inhibition protects against renal disease and other manifestations of autoimmune disease in MRL/lpr mice," Journal of Immunology, vol. 180, no. 2, pp. 1231-1238, 2008.

[22] L. Bao, M. Haas, D. M. Kraus et al., "Administration of a soluble recombinant complement $\mathrm{C} 3$ inhibitor protects against renal disease in MRL/lpr mice," Journal of the American Society of Nephrology, vol. 14, no. 3, pp. 670-679, 2003.

[23] M. M. Markiewski and J. D. Lambris, "The role of complement in inflammatory diseases from behind the scenes into the spotlight," American Journal of Pathology, vol. 171, no. 3, pp. 715-727, 2007.

[24] M. V. Carroll and R. B. Sim, "Complement in health and disease," Advanced Drug Delivery Reviews, vol. 63, pp. 965-975, 2011.

[25] S. Nasiri, M. Karimifar, Z. S. Bonakdar, and M. Salesi, "Correlation of ESR, C3, C4, anti-DNA and lupus activity based on British isles lupus assessment group index in patients of rheumatology clinic," Rheumatology International, vol. 30, no. 12, pp. 1605-1609, 2010.

[26] C. C. Liu, A. H. Kao, D. M. Hawkins et al., "Lymphocytebound complement activation products as biomarkers for diagnosis of systemic lupus erythematosus," Clinical and Translational Science, vol. 2, no. 4, pp. 300-308, 2009.

[27] J. C. Crispín, S. N. C. Liossis, K. Kis-Toth et al., "Pathogenesis of human systemic lupus erythematosus: recent advances," Trends in Molecular Medicine, vol. 16, no. 2, pp. 47-57, 2010.

[28] C. Edgerton, J. C. Crispín, C. M. Moratz et al., "IL-17 producing CD4+ $\mathrm{T}$ cells mediate accelerated ischemia/reperfusioninduced injury in autoimmunity-prone mice," Clinical Immunology, vol. 130, no. 3, pp. 313-321, 2009.

[29] G. Karpel-Massler, S. D. Fleming, M. Kirschfink, and G. C. Tsokos, "Human C1 esterase inhibitor attenuates murine mesenteric ischemia/reperfusion induced local organ injury," Journal of Surgical Research, vol. 115, no. 2, pp. 247-256, 2003.

[30] S. D. Fleming, T. Shea-Donohue, J. M. Guthridge et al., "Mice deficient in complement receptors 1 and 2 lack a tissue injuryinducing subset of the natural antibody repertoire," Journal of Immunology, vol. 169, no. 4, pp. 2126-2133, 2002.

[31] M. Ling, S. J. Piddlesden, and B. P. Morgan, "A component of the medicinal herb ephedra blocks activation in the classical and alternative pathways of complement," Clinical and Experimental Immunology, vol. 102, no. 3, pp. 582-588, 1995.

[32] E. I. B. Peerschke, B. Andemariam, W. Yin, and J. B. Bussel, "Complement activation on platelets correlates with a 
decrease in circulating immature platelets in patients with immune thrombocytopenic purpura," British Journal of Haematology, vol. 148, no. 4, pp. 638-645, 2010.

[33] E. I. B. Peerschke, W. Yin, D. R. Alpert, R. A. S. Roubey, J. E. Salmon, and B. Ghebrehiwet, "Serum complement activation on heterologous platelets is associated with arterial thrombosis in patients with systemic lupus erythematosus and antiphospholipid antibodies," Lupus, vol. 18, no. 6, pp. 530538, 2009.

[34] M. Huber-Lang, J. V. Sarma, F. S. Zetoune et al., "Generation of $\mathrm{C} 5 \mathrm{a}$ in the absence of $\mathrm{C} 3$ : a new complement activation pathway," Nature Medicine, vol. 12, no. 6, pp. 682-687, 2006.

[35] C. T. Esmon, "The interactions between inflammation and coagulation," British Journal of Haematology, vol. 131, no. 4, pp. 417-430, 2005.

[36] A. Nayak, J. Ferluga, A. G. Tsolaki, and U. Kishore, "The non-classical functions of the classical complement pathway recognition subcomponent C1q," Immunology Letters, vol. 131, no. 2, pp. 139-150, 2010.

[37] K. Ritis, M. Doumas, D. Mastellos et al., "A novel C5a receptortissue factor cross-talk in neutrophils links innate immunity to coagulation pathways," Journal of Immunology, vol. 177, no. 7, pp. 4794-4802, 2006.

[38] K. Oikonomopoulou, D. Ricklin, P. A. Ward, and J. D. Lambris, "Interactions between coagulation and complementtheir role in inflammation," Seminars in Immunopathology, vol. 34, no. 1, pp. 151-165, 2012.

[39] F. Bossi, E. I. Peerschke, B. Ghebrehiwet, and F. Tedesco, "Cross-talk between the complement and the kinin system in vascular permeability," Immunology Letters, vol. 140, pp. 7-13, 2011.

[40] C. T. Esmon, J. Xu, and F. Lupu, "Innate immunity and coagulation," Journal of Thrombosis and Haemostasis, vol. 9, supplement 1, pp. 182-188, 2011.

[41] Z. Shariat-Madar, F. Mahdi, and A. H. Schmaier, "Assembly and activation of the plasma kallikrein/kinin system: a new interpretation," International Immunopharmacology, vol. 2, no. 13-14, pp. 1841-1849, 2002. 


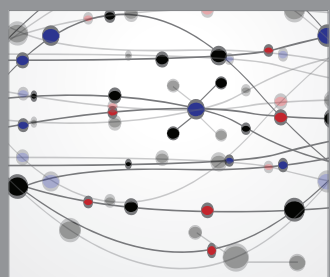

The Scientific World Journal
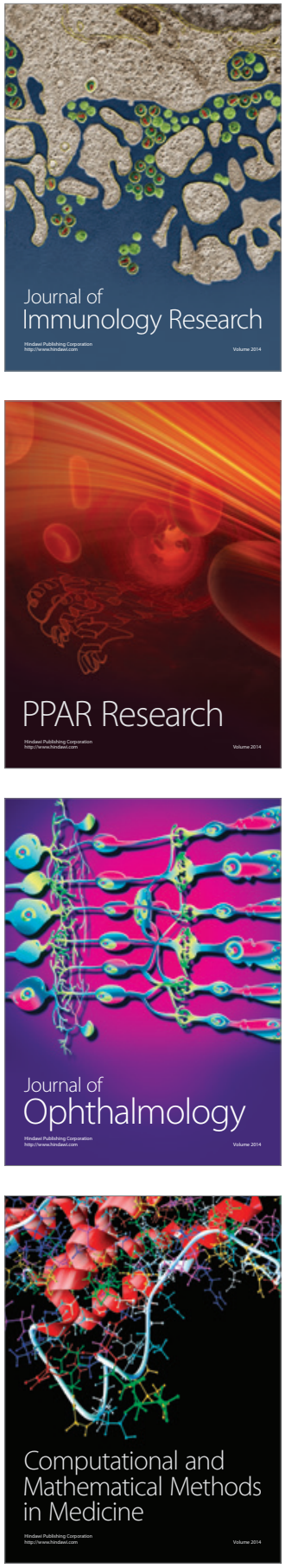

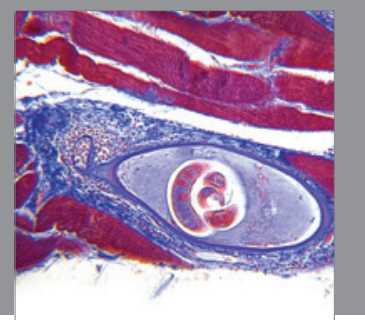

Gastroenterology

Research and Practice
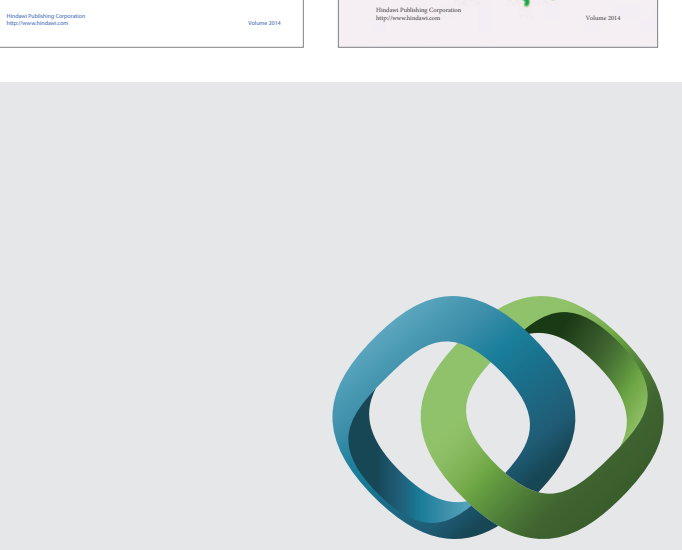

\section{Hindawi}

Submit your manuscripts at

http://www.hindawi.com
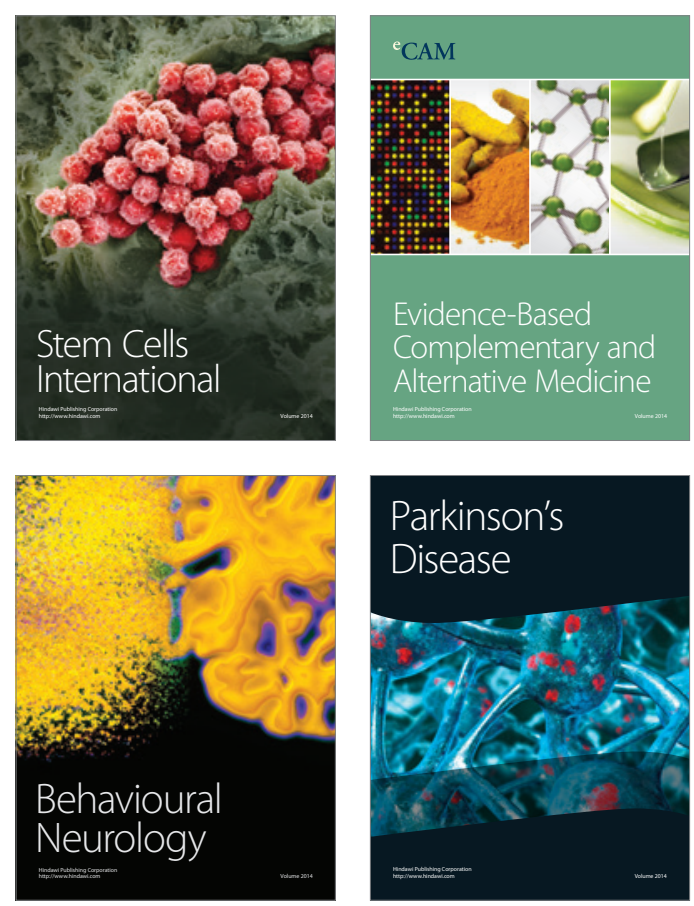

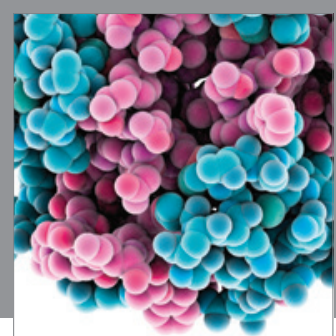

Journal of
Diabetes Research

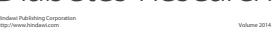

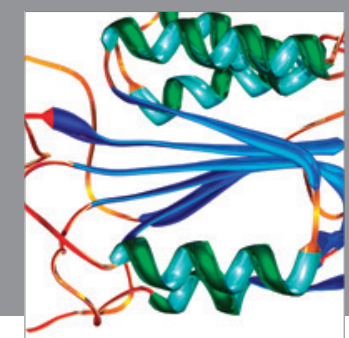

Disease Markers
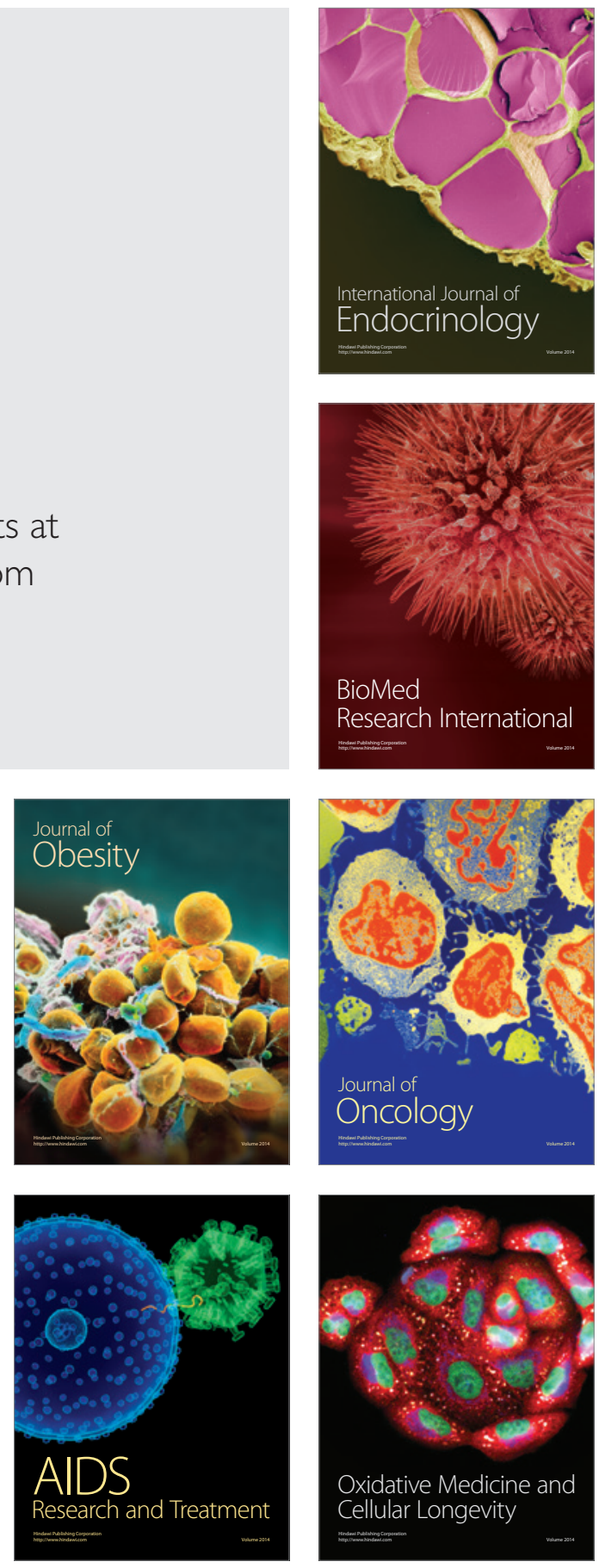